\title{
Inferences from the dark sky: Olbers' paradox revisited
}

\author{
Mauro Arpino \\ Civico Planetario "Ulrico Hoepli" \\ Corso Venezia 57, 20121 Milano, Italy. \\ email: arpino@tiscali.it \\ Fabio Scardigli卬 \\ Institute for Theoretical Physics, University of Bern, \\ Sidlerstrasse 5, 3012 Bern, Switzerland. \\ email: fabio@itp.unibe.ch
}

\begin{abstract}
The classical formulation of "Olbers' Paradox" consists in looking for an explanation of the fact that the sky at night is dark. We use the experimental datum of the nocturnal darkness in order to put constraints on a newtonian cosmological model. We infer then that the Stellar System in such a model should have had an origin at a finite time in the past.
\end{abstract}

PACS 98.80 - Cosmology.

\section{Introduction}

The observation that the sky at night is dark seems to be in conflict with the idea of an infinite Universe. If the Universe is infinite and contains an unlimited number of stars, our line of sight should meet a star in every direction we observe. Therefore, there should be no apparent gaps between stars on the celestial sphere. Moreover, repeating an argument originally attributed to de Cheseaux (1718-1751) [1], we note that, in an infinitely populated Universe, the number of stars contained in a spherical shell of radius $r$ is $4 \pi n r^{2} d r$, where $n$ is the number of stars per unit volume. If $L$ is the absolute luminosity of a star, the intensity of light at a distance $r$ is $I=L / 4 \pi r^{2}$. Hence the generic shell produces a lighting (in the centre of the shell) equal to $n L d r$. Since there is an

\footnotetext{
${ }^{1}$ Corresponding author: Fabio Scardigli.

Address for all the correspondence: Via Europa 20 - 20097 San Donato Milanese - Milano, ITALY.
} 
infinite number of shells, the lighting, in every point of the Universe, should be infinite!

But the sky at night is dark. This apparent contradiction has been named Olbers' Paradox, following the publication in 1823 of H. W. Olbers' paper "On the Transparency of Space" [2]. However, the first assertions about the conflict between the dimensions of the Universe and the nocturnal darkness can be traced back to Digges (1576), Kepler (1610) and Halley (1721) [3].

Many explanations were given during the centuries in attempts to resolve the paradox. Olbers suggested the presence of interstellar dust obscuring the farthest stars, but simple thermodynamical considerations by J. Herschel (1848) [soon ruled out this explanation. Others invoked hypotheses that required a hierarchic distribution of stars [5]. In 1917 Shapley suggested the idea of an "island-universe", that is a limited spatial extension of the stellar system, as a resolution of the paradox. After the discovery of the expansion of the Universe, the redshift was proposed as the fundamental mechanism necessary to "cut" the radiation coming from the farthest galaxies, hence assuring the darkness of night.

Harrison [6] suggested that the paradox does not exist even in the context of a classical Universe, in the framework of which it was conceived, because there are too few stars and their lifetimes are too short. In other words the Universe does not contain enough energy to produce a bright sky. Nevertheless, in current literature there are still many misunderstandings about the paradox. It is often explained using the redshift, which, on the contrary, does not have a relevant role [7]. In fact detailed calculations show that in our Universe the luminosity of the sky is determined to order of magnitude by the lifetime of the galaxies, and only affected by a factor of two by the expansion of the Universe (see [8], which also gives a survey of the recent literature on the subject).

The classical formulation of Olbers' Paradox tries to explain the fact that the night is dark. In contrast, in the present paper, we use this observed fact to put some constraints on a simple euclidean-newtonian cosmological model. We then draw several necessary conclusions about the origin of the Universe (i.e. the Stellar System) at a finite time in the past.

\section{The cosmological model}

The model of space-time used here is the classical euclidean-newtonian one. The Universe is a euclidean manifold $\Re \times \Re^{3}$, with a euclidean metric on $\Re^{3}$. The time we use is the absolute time of Newton. The generic event is singled out by orthogonal cartesian coordinates $(t, \vec{x}) \in \Re \times \Re^{3}$. Therefore space has an infinite extension and is isotropic, homogeneous and flat in every region and so it coincides with Newton's absolute space. The simultaneity we refer to is the newtonian absolute simultaneity. The light signals propagate on euclidean straight lines and the speed of light is finite and equal to $c$.

In this model, all matter is in the form of stars. They are distributed in a homogeneous and isotropic way in the regions they fill. Therefore, there 
are no bunches of stars, such as clusters, galaxies, etc. The number of stars per unit volume does not change with time. This hypothesis is equivalent to assuming that the lifetime of a "virtual star" is, a priori, unlimited. From an astrophysical point of view, it means that a star does not have chemical, nuclear or gravitational evolution. Of course, based on current knowledge of stellar evolution, this is not true. Yet, from the cosmological point of view, this idea is reasonable if we admit an uninterrupted sequence of stellar generations. For every star that dies, a new one comes up, so the density of "active" stars does not change over time. As we will see, the comparison of the model with the experimental evidence of the dark at night will allow us to decide between the hypothesis of an "eternal" stellar system (i.e. an infinite number of generations) or the hypothesis of a stellar system born at a finite time in the past.

Of course, the adopted model does not describe any of the main properties of the contemporary models (such as expansion, curvature, red shift, etc.). Yet, we have chosen this model for two reasons. The first is its metric simplicity, which will let us introduce the concept of "lookout limit" without mathematical complexity. The second reason is that this model coincides with the model of classical newtonian space-time, historically adopted by Halley, Olbers, Shapley, until the Einstein paper on cosmology (1917) [9]. Finally, we want to note that the closer the global curvature is to zero, the more the euclidean model approximates the curved space-time.

\section{Lookout limit and background radiation en- ergy density}

Let us think of the stars as uniformly distributed in space. We then suppose that each star occupies an average volume $V$. Furthermore, each star has a section of area $\sigma$. Thus, the probability that our line of sight meets a star surface is

$$
p=\frac{\sigma}{V^{2 / 3}} .
$$

Our line of sight is certain to meet a star, if we observe a number $N$ of elementary volumes $V$, lined up in sequence, equal to

$$
N=\frac{V^{2 / 3}}{\sigma} .
$$

This is true, of course, if the stars are distributed completely at random in their respective volumes.

The distance reached by the line of sight before meeting a star surface is therefore

$$
\delta=V^{1 / 3} \frac{V^{2 / 3}}{\sigma}=\frac{V}{\sigma} .
$$


We call this distance lookout limit, after Harrison. It is well known that this quantity coincides with the mean free path $\lambda$ of a photon [10; in fact $V=1 / n$, where $n$ is the number of stars per unit volume and

$$
\lambda:=\frac{1}{\sigma n}=\frac{V}{\sigma}=\delta .
$$

Following Harrison and Kelvin [11], we will derive an expression for the fraction of sky covered by stellar discs, and then a formula for the radiation energy density (at the point of the observer) due to the whole stellar system.

Let us consider a spherical shell of radius $r$ and thickness $d r$. Let $\Omega(r)$ be the solid angle free of stellar discs up to the shell $r$. Let $\Delta \Omega(r)$ be the solid angle intercepted by the stars present on the shell $r$. The fraction of solid angle intercepted by stars on the shell $r$ is 10

$$
\frac{\Delta \Omega}{\Omega}=\frac{\text { total stellar area }}{\text { total shell area }}=\frac{-n \sigma 4 \pi r^{2} d r}{4 \pi r^{2}}=-n \sigma d r .
$$

After integration, we obtain an expression for the solid angle still free of stellar discs after a radial path $r$

$$
\Omega(r)=\Omega_{0} e^{-n \sigma r}
$$

where $\Omega_{0} \equiv 4 \pi$.

The solid angle intercepted by the stellar surfaces after a path $r$ is therefore

$$
\Omega_{0}-\Omega(r)=\Omega_{0}\left(1-e^{-n \sigma r}\right) .
$$

Hence the fraction of intercepted solid angle is

$$
\alpha=\frac{\Omega_{0}-\Omega(r)}{\Omega_{0}}=1-e^{-n \sigma r} .
$$

$\alpha$ is the fraction of sky covered by stellar discs out to the radius $r$. We note that if the stellar distribution is spatially unlimited, we can push $r \rightarrow \infty$, and then $\alpha \rightarrow 1$, i.e. the whole sky is covered by stars.

Also important is the relation

$$
u(r)=4 u^{*}\left(1-e^{-n \sigma r}\right),
$$

where $u(r)$ is the radiation energy density at the centre of a sphere of radius $r$, due to all the stars contained in the sphere, and $u^{*}$ is the radiation energy density at the surface of a star.

This relation can be derived as follows.

In a time $d t$ a star emits an energy $d E=L d t$, where $L$ is the star's luminosity. This energy spreads (in the time $d t$ ) on a volume $4 \sigma c d t$, where $4 \sigma$ is the surface area of a star with a section of area $\sigma$. Therefore we can write $d E=4 u^{*} \sigma c d t$, where $u^{*}=L /(4 \sigma c)$ is the radiation energy density at the surface of the star. 
The radiated energy $d E$ travels until it reaches a distance $r$ from the star. Then, in the same time $d t$ as before, it spreads on a volume $4 \pi r^{2} c d t$. Therefore the radiation energy density at a distance $r$ from the source is

$$
u=\frac{d E}{4 \pi r^{2} c d t}=\frac{L d t}{4 \pi r^{2} c d t}=\frac{u^{*} \sigma}{\pi r^{2}} .
$$

Now, the number of stars on a shell of radius $r$ is

$$
4 \pi r^{2} n d r
$$

and the fraction of free sky, i.e. not covered by stellar discs at a distance $r$ from the observer, is (see eqs. (6), (8))

$$
\frac{\Omega(r)}{\Omega_{0}}=e^{-n \sigma r} .
$$

Therefore the number of stars not obscured by other stars, i.e. visible at the centre of the shell, is

$$
4 \pi r^{2} n d r e^{-n \sigma r},
$$

and the contribution to the radiation energy density at the centre of the shell due to the stars on the shell is

$$
d u=4 \pi r^{2} n d r e^{-n \sigma r} \frac{u^{*} \sigma}{\pi r^{2}}=4 u^{*} \sigma n e^{-n \sigma r} d r .
$$

Integrating this relation between 0 and $r$, we obtain formula (9).

Hence $u$ represents the energy density of the background sky, or what is today called extragalactic background light. We note that $u=4 u^{*}$ in every star distribution extending to $r \gg \lambda=1 / n \sigma$. For an infinite spatial extension of the

stellar system, the background sky energy density must be of the same order of that on the surface of a star.

The experimental condition of dark sky can be expressed in this context as

$$
u \ll u^{*} .
$$

We note that the presence of the factor $e^{-n \sigma r}$ (which accounts for the mutual absorption of light by stars in the Universe) produces a lowering of lighting at any point in space from $\infty$ to $4 u^{*}$.

\section{Study of the model}

We are now able to examine all the cases suggested by the proposed model using the concept of lookout limit, having adopted absolute newtonian space and time, and having supposed that they both have an infinite extension.

For the stellar system, we have the two following possibilities: 
I) The distribution of the stellar system is spatially infinite.

II) The distribution of the stellar system is spatially finite. We suppose then that it has a spherical symmetry with a radius $R$.

In case I) we can distinguish two subcases:

Ia) The stellar system has existed from an infinite time in the past.

Ib) The stellar system was "turned on" (all the stars together and simultaneously) at a time $t_{0}=-T$ in the past $(T>0)$ (we take $t=0$ as the present time).

In case Ia) it is evident that the sky at night must be luminous. In fact every line of sight should necessarily intercept, sooner or later, a star surface $(\alpha=1)$. Therefore the celestial sphere must appear luminous, completely filled up by stellar discs, without dark spaces among stellar discs $\left(u=4 u^{*}\right)$. Case Ia), a spatially infinite Universe, is the model adopted by Halley and Olbers, on the grounds of newtonian considerations. It is in evident conflict with observation, a conflict historically known as Olbers' Paradox.

In case $\mathrm{Ib}$ ), only the radiation of stars contained in a sphere of radius $h=c T$ (cosmological horizon) can reach us. The radius of this sphere must be compared with the value of the lookout limit $\lambda$. We can distinguish the following subcases.

Ib1) If $\lambda>c T$ then the night sky could turn out to be "dark", that is

$$
u(c T)<u(\lambda)=4\left(1-\frac{1}{e}\right) u^{*} .
$$

In fact, the lines of sight can extend outward to a distance of $r=\lambda$, beyond the "border" $r=c T$ of the stellar system. This situation is consistent with the observed evidence.

Ib2) If $\lambda<c T$ then the sky at night must be "luminous", that is

$$
u(c T)>4\left(1-\frac{1}{e}\right) u^{*} .
$$

Of course this possibility is excluded by the experimental evidence.

Let us now study case II). We have two sub-cases here as well:

IIa) The stellar system has existed from an infinite time in the past $\left(t_{0}=-\infty\right)$;

IIb) The stellar system was "turned on" at a time $t_{0}=-T$ in the past.

In case IIa) we can compare $\lambda$ with the radius $\mathrm{R}$ of the stellar system: 
IIa1) If $\lambda>R$ then the night sky could be "dark", i.e. $u(R)<4(1-1 / e) u^{*}$.

IIa2) If $\lambda<R$ then the night sky should be "luminous", i.e. $u(R)>4(1-1 / e) u^{*}$.

The first case is in apparent agreement with the empirical evidence. Yet we observe that the assumptions " $\mathrm{R}$ finite" and " $t_{0}=-\infty$ " create problems in the dynamical stability of the system. From the point of view of newtonian mechanics, a homogeneous sphere, with a finite radius and a negligible angular momentum, made of particles interacting only by gravity, has to collapse inwards upon its geometrical centre in a finite time. For such dynamical reasons case IIa1) offers little of physical interest and can be ruled out.

In case IIb), stars were born at a time $t_{0}=-T$ in the past. The quantities to be compared are now the lookout limit $\lambda$, the radius $R$ of the stellar system, and the horizon $h=c T$ of the observable spherical region of the stellar system. We have therefore $6(=3$ !) different cases. Their analysis is summarized by the following table.

$$
\begin{aligned}
& \text { IIb1) } c T<\lambda<R \Rightarrow \quad\left(\text { dark sky: } u(c T)<4(1-1 / e) u^{*}\right) \\
& \text { IIb2) } \left.\lambda<c T<R \Rightarrow \text { (luminous sky: } u(c T)>4(1-1 / e) u^{*}\right) \\
& \text { IIb3) } \left.\lambda<R<c T \Rightarrow \text { (luminous sky: } u(R)>4(1-1 / e) u^{*}\right) \\
& \operatorname{IIb} 4) R<\lambda<c T \Rightarrow \quad\left(\text { dark sky: } u(R)<4(1-1 / e) u^{*}\right) \\
& \operatorname{IIb5)} c T<R<\lambda \Rightarrow \quad\left(\text { dark sky: } u(c T)<4(1-1 / e) u^{*}\right) \\
& \text { IIb6) } R<c T<\lambda \Rightarrow \quad\left(\text { dark sky: } u(R)<4(1-1 / e) u^{*}\right) .
\end{aligned}
$$

We should point out that in cases IIb) also, with $R<\infty, t_{0}=-T$, there could be problems of dynamical instability. We can say that the collapse of the spherical stellar system is unavoidable; nevertheless we can think that the collapse is not observable if we suppose that it is very slow compared with the cosmic time scale $(\mathrm{T})$ (speed of collapse $\ll c$ ).

It is interesting to note that Newton himself investigated the problem of the dynamical stability of a finite spherical system of stars. He knew that such a system should have collapsed after a finite time inwards upon its centre of mass. But, strangely enough, he avoided calculating the time for gravitational collapse. Only in 1902, did Lord Kelvin complete the first cosmological calculus 
on the collapse time of the whole stellar system 11]. He found that the collapse time does not depend on the initial size of the system. Instead, it depends only on its initial density

$$
t_{k}=\left(\frac{3 \pi}{32 G \rho_{0}}\right)^{1 / 2} .
$$

Assuming an average interstellar distance of 1 pc and a mean mass of 1 solar mass, Kelvin obtained a time for the collapse equal to $20 \times 10^{6}$ years. Yet, Kelvin used classical arguments: he ignored the relativistic limit of the speed of light and therefore he obtained such a short collapse time that it was not realistic if compared with the cosmic time scale. If we take into account the limiting speed of light $c$, then the collapse time cannot be less than the radius of the universe divided by $c$, because the various regions will collapse, at the fastest, with a speed equal to $c$.

\section{Conclusions}

The comparison of the cases presented in the above section with the experimental evidence of the dark sky at night, allows us to eliminate cases Ia, Ib2, IIa2, IIb2, IIb3. They produce a luminous sky at night. Only cases Ib1, IIb1, IIb4, IIb5, IIb6 are physically admissible, because they are consistent with the experimental evidence. What property do they share?

The main consideration is that all these cases imply the birth of the Stellar System at a finite time in the past. We note that our model, together with the evidence of the dark sky, is not able to discriminate between a Universe with a finite radius and one with an infinite radius.

Therefore the conclusion that can be drawn from our model is that the experimental evidence of the dark sky implies that the Stellar System was born at a finite time in the past. This important observation was not formulated by Olbers or others (Halley, Kelvin, etc.). Olbers would have been able to infer, from the observed fact of a dark night sky, the birth of the cosmos at a finite time in the past. This could happen because the cosmological model adopted by Olbers (and more or less explicitly by all the scientists until Einstein) is the newtonian one described in section 2. The lookout limit introduced by Harrison is a useful concept which has allowed us to obtain a deeper and more effective understanding of the model.

We want to emphasize that other authors have focused particularly on the luminous lifetime of single stars. But this has prevented them from reaching the relevant conclusions about the lifetime of the whole stellar system, i.e. the Universe. The main hypothesis that allowed us to obtain information about the lifetime of the Universe (the Stellar System) is the one of the "virtual star", i.e.

a fictitious luminous source that, in principle, has an infinite life. Successive generations of real stars can be modelled as a single virtual star without an a priori fixed lifetime. The check with the observational evidence $\left(u \ll u^{*}\right)$ 
determines, at this point, the lifetime of the virtual star, i.e. of the whole Stellar System. In other words, we could say that classical physics (without hypotheses on nuclear reactions and expansion of the Universe) is able to explain the nocturnal darkness of the sky only by requiring a finite lifetime of the Stellar System.

Finally we want to observe that several authors (e.g. Weinberg) state that in a Big Bang cosmology there is no paradox because the contribution of the various stellar generations is cut at a finite time in the past [12]. This is not always true from our point of view, because we have seen that we can have a "Big Bang cosmology" (in the newtonian sense, namely that the stellar system was "turned on" in the past), which nonetheless produces a luminous sky (cases Ib2, IIb2, IIb3). In conclusion, Olbers would not have been able to infer the expansion of the Universe from the observation of the dark sky at night, as several authors 13] seem to suggest. On the contrary, Olbers and other authors (from the 18th to the early 20th centuries) would have been able to assert that the Universe has had a temporal origin in the past.

\section{Acknowledgements}

The authors wish to thank the anonymous referee for useful comments, T.J. Lindsey for style suggestions, and the nice Irish Pub "Crazy Patrick", where this paper was conceived.

\section{References}

[1] J.P.L. de Cheseaux, Traite' de la Comete (M.M.Bousequet, Lausanne, 1744).

[2] H.W.Olbers, Über die Durchsichtigkeit des Weltraumes, Astronomisches Jahrbuch für das Jahr 1826, C.F.E. Spaethen, Berlin (1823); Engl. transl. On the Transparency of Space, Edinburgh New Philosophical Journal, 1, 141 (1826).

[3] E.R.Harrison, Olbers' Paradox, Nature, 204, 271-272 (1964).

[4] J.F.W.Herschel, Humboldt's Kosmos, Edinburgh Rev. 87, 170 (1848).

[5] S.L. Jaki, Olbers', Halley's, or whose Paradox?, Am.J.Phys. 35, 200-210 (1967).

[6] E.R.Harrison, Why the sky is dark at night, Phys.Today 27(2), 30-36 (1974).

[7] E.R.Harrison, The dark night sky paradox, Am.J.Phys. 45(2), 119-124 (1977).

E.R.Harrison, Cosmology. The Science of the Universe (Cambridge U.P., Cambridge, 1981). 
[8] P.S.Wesson, K.Valle, R.Stabell, The extragalactic background light and a definitive resolution of the Olbers' paradox, Astrophys.J. 317, 601-606 (1987). P.S.Wesson, Olbers' paradox and the spectral intensity of extragalactic background light, Astrophys.J. 367, 399-406 (1991).

[9] A. Einstein, Kosmologische Betrachtungen zur allgemeinen Relativitaetstheorie, Sitz. Preuss. Akad. Wiss., 142-152 (1917).

[10] H.A.Enge, M.R.Wehr, J.A.Richards, Introduction to Atomic Physics (Addison-Wesley, Reading-Mass. 1973).

[11] E.R.Harrison, Kelvin on a old, celebrated hypothesis, Nature, 322, 417-418 (1986).

[12] S.Weinberg, Gravitation and Cosmology (J.Wiley and S., New York, 1972).

[13] H.Bondi, Cosmology (Cambridge U.P., Cambridge, 1952).

D.W.Sciama, The Unity of the Universe (Faber and Faber, London, 1959). 